\title{
REVIEW
}

\section{The concept of treatment-free remission in chronic myeloid leukemia}

\author{
S Saußele ${ }^{1}$, J Richter ${ }^{2}$, A Hochhaus ${ }^{3}$ and F-X Mahon ${ }^{4}$
}

The advent of tyrosine kinase inhibitors (TKI) into the management of patients with chronic myeloid leukemia (CML) has profoundly improved prognosis. Survival of responders is approaching that of the general population but lifelong treatment is still recommended. In several trials, TKI treatment has been stopped successfully in approximately half of the patients with deep molecular response. This has prompted the development of a new concept in the evaluation of CML patients known as 'treatmentfree remission'. The future in CML treatment will be to define criteria for the safe and most promising discontinuation of TKI on one hand, and, on the other, to increase the number of patients available for such an attempt. Until safe criteria have been defined, discontinuation of therapy is still experimental and should be restricted to clinical trials or registries. This review will provide an overview of current knowledge as well as an outlook on future challenges.

Leukemia (2016) 30, 1638-1647; doi:10.1038/leu.2016.115

\section{INTRODUCTION}

In chronic myeloid leukemia (CML), treatment with tyrosine kinase inhibitors (TKI) directed at the pathogenetic structure, the abnormal BCR-ABL fusion tyrosine kinase, can achieve durable cytogenetic and molecular remissions (MRs) and substantially improve survival in the majority of patients. ${ }^{1,2}$ There is reasonable expectation not only of improving survival further but of curing the disease since a high percentage of patients reach deep molecular response. ${ }^{3,4}$ The most recent recommendations of the National Comprehensive Cancer Network (NCCN) and the European LeukemiaNet (ELN) for CML propose continuation of TKI treatment indefinitely in all responding patients. ${ }^{5,6}$ Current drawbacks of lifelong treatment include expected and unexpected side-effects, impairment of quality of life (QoL) for many patients ${ }^{7}$ and the price of the drugs.

Recent studies have demonstrated a considerable proportion of patients suffering from TKI side effects ${ }^{7}$ and previous unknown toxicities. ${ }^{8-10}$ Adverse drug reactions such as arterial hypertension, pleural effusion and vascular events were unexpected and reported after approval of the TKI. For imatinib, the first TKI employed in CML, an association has been established between treatment and side effects that impair QoL especially in younger patients. ${ }^{7,11,12}$ For newer TKIs, data on QoL are scarce. ${ }^{13}$

Improved survival rates in CML lead to increasing prevalence of the disease. With more than $400000 \mathrm{CML}$ patients expected in Europe by the year 2050, CML is set to become an important chronic non-communicable disease. The cost of treatment per patient is currently estimated at $\sim 30000-40000 €$ per year in most European countries. In view of recommended lifelong treatment, ${ }^{6}$ this represents a significant burden on health care systems, ${ }^{14}$ even though costs are expected to decline when generic imatinib becomes available in most countries.
Stopping TKI treatment in a substantial percentage of patients would be an innovative and, importantly, a cost-effective way to optimize available therapy concepts. Several studies have shown that $\sim 40 \%$ of patients in stable deep molecular response stay in treatment-free remission (TFR) after stopping first-line treatment. In general, after molecular relapse, patients remain sensitive to a TKI and regain molecular response.

However, definitions of molecular response have changed over time. The actual definitions differentiate several categories of $M R^{15,16}$ according to the level of detectable BCR-ABL transcripts and the sensitivity of the individual assay which was not the case when the first discontinuation studies were initiated.

This review aims to provide an overview of current data, to critically discuss results of hitherto published studies and the remaining open questions, and finally to provide an outlook on next steps and future perspectives.

\section{STOP-THERAPY ATTEMPTS}

Even before the advent of TKI in CML therapy, successful discontinuation of interferon-alpha (IFN) treatment in complete cytogenetic remission had been described. ${ }^{17}$ During this era, quantitative reverse transcription (qRT)-PCR techniques were not available or cumbersome and not standardized for the sensitivity of the individual test. However, a minority of patients discontinued IFN-alpha therapy and remained in remission. ${ }^{18}$

Promising results from case reports and small studies under TKI treatment ${ }^{19-21}$ encouraged the launch of stopping trials. In a pilot study, ${ }^{22}$ out of 12 patients with CML who discontinued imatinib, 6 maintained complete molecular response (CMR) for at least 2 years. In that study and at that time, CMR was defined by undetectable BCR-ABL transcripts; the sensitivity of the PCR assay was retrospectively defined between 4.5 and 5 log. After a median

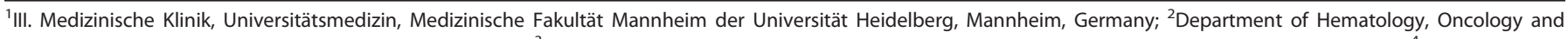

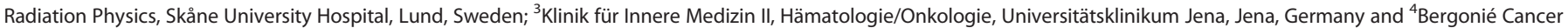

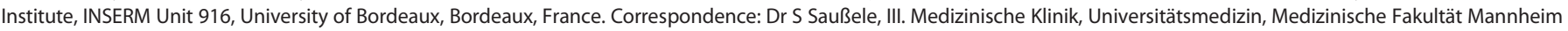
der Universität Heidelberg, Pettenkoferstrasse 22, 68169 Mannheim, Germany.

E-mail: susanne.saussele@medma.uni-heidelberg.de

Received 22 February 2016; revised 22 March 2016; accepted 30 March 2016; accepted article preview online 2 May 2016 ; advance online publication, 3 June 2016 
follow-up of 18 months, $50 \%$ of patients remained off therapy without confirmed reappearance of BCR-ABL transcripts in peripheral blood. Updated results confirmed that $50 \%$ of patients off therapy had an undetectable level of BCR-ABL transcripts after a median follow-up of 7.5 years (range, $4.4-8.4$ years). ${ }^{23}$ This pilot study provided proof of concept that imatinib discontinuation could be achieved in selected CML patients.

The multicenter stopping imatinib (STIM) study ${ }^{24}$ confirmed this approach in a larger cohort of patients. One hundred chronic phase (CP) CML patients on first-line imatinib therapy with identical entry criteria were included prospectively. The main requirement for study entry was consistently undetectable peripheral blood BCR-ABL transcripts for at least 2 years. Molecular relapse, which was arbitrarily defined as two positive quantitative Real-Time PCR (qRT-PCR) results within a month showing a significant rise (1log) in $B C R-A B L$ transcript numbers, triggered the restart of imatinib treatment. An interim analysis yielded promising results with a 12-month molecular relapse-free survival rate of $41 \% .{ }^{24} \mathrm{~A}$ recent update of that study confirmed that cumulative incidence of molecular relapses at 65 months was $61 \%(5 \%$, confidence interval (Cl) 52-70) and three cases of late relapse were observed at months 19,20 and 22 , respectively. ${ }^{25}$ The probability of a molecular relapse after the first 6 months period is only $\sim 10 \%$. Stopping TKI intake did not harm patients; most patients who experienced molecular relapse did so within 6 months of imatinib cessation and remained responsive to re-treatment with imatinib as observed in the pilot study.

The CML8 (TWISTER) study of the Australasian Leukaemia and Lymphoma Group (ALLG), which used entry criteria very similar to STIM (patients treated on imatinib for more than 2 years and PCR negativity with a sensitivity of $4.5 \mathrm{log}$ ), confirmed these results. ${ }^{26-28}$ The median follow-up of the TWISTER Study was 42 months. The estimated proportion of stable TFR was $47.1 \%$ at 24 months. Most relapses occurred within 6 months after stopping imatinib, and no relapses beyond 27 months were observed.

A retrospective survey in Japan identified 50 patients who had discontinued imatinib for at least 6 months, 43 of whom were analysed. Molecular recurrence was detected in 19 patients, and the molecular relapse-free survival rate following imatinib discontinuation was estimated to be $47 \%{ }^{29}$

Although under close molecular monitoring, it was observed that low levels of residual disease after TKI withdrawal did not automatically indicate CML relapse and did not preclude the possibility of remaining treatment-free. To prove this principle, a French multicentre observational study (according to stop imatinib (A-STIM)) was conducted in 80 patients with $\mathrm{CP}-\mathrm{CML}$ who had stopped imatinib after sustained CMR of 2 years, using the same definition of CMR as in the STIM study. ${ }^{30}$ However, the criteria for stopping were less stringent than in the STIM study, and patients with positive low-level PCR results corresponding to $\mathrm{MR}^{4.5}$ were also considered eligible. ${ }^{30}$ Molecular relapse was also less stringently defined: loss of major molecular response (MMR, $\mathrm{BCR}-\mathrm{ABL} \leqslant 0.1 \%$ on the international scale (IS)) at any time was chosen for triggering re-treatment. The median follow-up after discontinuation was 31 months (range, 8-92 months). Twentynine patients (36\%) lost MMR after a median of 4 months off therapy (range, 2-17 months). Cumulative incidence of MMR loss was $35 \%(95 \% \mathrm{Cl}, 25-46 \%)$ at 12 months, and $36 \%$ at 24 months $(95 \% \mathrm{Cl}, 24-48 \%)$ with a plateau after 24 months. Fluctuation of BCR-ABL transcript levels below the MMR threshold was observed in $31 \%$ of patients after imatinib discontinuation. TFR was estimated $\sim 37 \%$ at 36 months when the STIM criteria were applied, that is, close to STIM or TWISTER results. The study was also safe as all patients were sensitive to re-treatment. The main conclusion drawn from the study was to use 'loss of MMR' as the more appropriate trigger for restarting treatment after TKI discontinuation than loss of deep molecular response or CMR.
Most recently, data of the ISAV (Imatinib Suspension and Validation) study were presented..$^{31}$ One hundred and twelve patients with at least 2-year imatinib treatment and at least 18 months undetectable transcripts in QRT-PCR were followed for a median of 21.6 months. Cumulative incidence of relapses was $52 \%$ after 36 months. Relapse was defined as loss of MMR (two consecutive positive PCRs with one result at least above $0.1 \%)$. This is the first study which used digital PCR in parallel with qRT-PCR, which produced more accurate prediction of relapse.

Several trials are ongoing: A STIM2 trial was launched in France in 2011 and is still in progress. This trial has included patients with CP-CML treated initially only with imatinib as a single agent with the same criteria as STIM, that is, sustained deep MR for at least 2 years. The criterion for molecular relapse was also similar. Preliminary results have been communicated on 127 recruited patients with a cumulative molecular relapse incidence of $46 \%$ (95\% Cl 38-56) at 24 months. $^{32}$

The largest study to date is the European Stop Kinase Inhibitor (EURO-SKI) trial of the ELN that is currently in progress. This study has recruited more than 800 patients. The EURO-SKI trial has been initiated in 68 centers in 11 countries. The criteria for discontinuation are less strict than in the STIM studies: the duration of TKI treatment before enrollment must be at least 3 years and no PCRresults $>0.01 \%$ (corresponding to $\mathrm{MR}^{4}$ ) within the last year before TKI discontinuation is allowed. The deep molecular response of 4 logs has to be confirmed in a standardized laboratory by the EUTOS program (European treatment and outcome study for $\mathrm{CML}$ ). The null hypothesis was that molecular relapse-free survival at 6 months would be $\leqslant 40 \%$. Already the results of the interim analysis of this study have demonstrated that the concept is feasible and practical. ${ }^{25}$ To date, out of the first 200 eligible patients with at least 6 months of follow-up, 123 patients have remained without molecular relapse defined as loss of MMR at any one point in time. Thus, the molecular relapse-free survival at 6 months was $61.5 \%,(95 \% \mathrm{Cl}(54.4 \% ; 68.3 \%))$, showing that the initial null hypothesis of this trial can be discarded $(P<0.0001){ }^{33}$

So far, only limited experience is available both of secondgeneration TKls and of patients who discontinued a second time after molecular relapse in a first treatment-free phase.

To address the feasibility of discontinuing nilotinib or dasatinib, an academic pilot study has begun in France. Preliminary results have been reported showing that among the 52 patients with at least 12 months of follow-up, 25 lost MMR; the probability to be in MMR at 12 months was $59.6 \%(95 \% \mathrm{Cl} 46-73)$. This study also has included patients with a prior failure to a first-line TKI. ${ }^{34}$

Recently, results of the Japanese Dasatinib Discontinuation (DADI) study evaluated TFR after suspension of dasatinib as second-line therapy or beyond were reported. With a median follow-up of 20 months, among 63 patients who attempted TFR, deep molecular response was maintained in 30 patients and 33 patients had molecular relapse; the probability of TFR was $49 \%$ and $48 \%$ at 6 and 12 months, respectively. ${ }^{35}$ All molecular relapses occurred within 7 months of stopping dasatinib therapy, and on treatment re-initiation (dasatinib, $n=32$; nilotinib, $n=1$ ) all 33 patients with molecular relapse regained deep molecular response within 6 months and the majority $(n=29)$ did so within 3 months.

Several other industry-sponsored trials are currently recruiting patients (DASFREE; Bristol-Myers Squibb), or are in follow-up (ENESTPath, ENESTFreedom, ENESTop, ENESTGoal; Novartis). ${ }^{36}$ Patients without deep MR on imatinib are switched to nilotinib on enrollment (induction phase). Following nilotinib induction therapy, patients enter the consolidation phase during which they continue nilotinib and are monitored for maintenance of the required MR. In ENESTFreedom and ENESTop, patients who have achieved $\mathrm{MR}^{4.5}$ on nilotinib enter the consolidation phase directly on enrollment. In all three studies, the required MR must be maintained through the consolidation phase (with molecular monitoring every 3 months) to be eligible to attempt TFR. Any 
patient with molecular relapse during the TFR phase must reinitiate therapy. Rates of successful TFR will be evaluated in each study. In DASFREE, a phase 2 study (Dasatinib Functional Cure; CA180-406 study), therapy discontinuation after at least 2 years of dasatinib treatment in patients with CML with stable deep molecular responses is evaluated. ${ }^{37}$ DESTINY (De-escalation and stopping treatment of imatinib, nilotinib or dasatinib in $(M L)$, is being conducted as an investigator-initiated trial in the United Kingdom. ${ }^{38}$ Patients in stable deep MR reduce the TKI dose to half of the standard dose for 12 months. For patients in stable remission at the end of the 12-month period, treatment is stopped. Patients will be followed for 2 years. All these trials use 'loss of MMR' as an endpoint.

So far, only one trial was published on patients discontinuing TKI treatment a second time. Sixteen patients in second CMR were treated for at least 1 year with imatinib after a first unsuccessful stop. $^{39}$ All patients were in MR within a median of 2.5 months (range, 1-8 months) and obtained a second CMR after imatinib reintroduction within a median of 6 months (range: 1-19 months) after this first attempt. Four patients (25\%) never lost their MMR and remained free of treatment with a median follow-up of 32 months (range, 15-53 months). Two trials are currently in the planning phase to investigate the concept of a second TKI-stop in patients failing a first cessation attempt, one including nilotinib as therapy before a second stop and the other one dasatinib.

\section{PROGNOSTIC FACTORS FOR SUCCESSFULLY STOPPING TKI IN CML}

Identifying predictive factors for successful discontinuation of TKI remains a key issue. In addition, a safe approach to stopping TKI treatment a second time must be defined. Several trials described above integrate the analysis of prognostic factors. In some studies, this is done as multivariate analysis and in some as univariate analysis. The problem of all hitherto published trials is the relatively small patient number. Therefore, the prognostic factors discussed in this section must be interpreted with caution and require further confirmation. To date, the prognostic impact of the duration of both prior treatment and prior MR as well as of the individual Sokal score at diagnosis on the chance to maintain TFR have been described.

\section{Treatment duration}

In the STIM study, which uses multivariate analysis and logistic regression at 8 months, imatinib therapy duration has been identified as an independent prognostic factor for prediction of molecular relapse after imatinib cessation. The rate of molecular relapse after discontinuation of imatinib was $53 \%$ of patients treated with imatinib for 50 months or more vs $83 \%$ of patients treated with imatinib for fewer than 50 months. ${ }^{24}$ In the EURO-SKI trial the median value of treatment duration (8 years) for the first 200 patients with at least 6 months of follow-up allows for a significant division of the population. However, the data has yet to be confirmed by multivariate analysis. ${ }^{33,40}$

\section{MR duration}

Different TKI discontinuation studies (Table 1) confirmed and suggested that the duration of response, especially the duration of deep MR before stopping, was important. Takahashi et al. reported a significant difference in the estimated molecular relapse-free survival rates at 5 years following discontinuation between patients in whom deep MR was sustained for more than 24 months before imatinib discontinuation and those sustaining a deep MR for $<24$ months (78 vs $15 \%, P=0.0002$ ). ${ }^{29}$

The validation of this criterion was reinforced using mathematical models confirming a biphasic decline of BCR-ABL transcript levels with a two-slope model of imatinib response: the $a$ slope corresponded to the rapid initial decrease in BCR-ABL transcript levels (cycling cells) after the start of treatment, and the $\beta$ slope corresponded to the longer-term BCR-ABL dynamics (less proliferative cells). ${ }^{41,42}$ Another model based on the biphasic decline of BCR-ABL transcript levels suggested that $31 \%$ of the patients would remain in deep MR after treatment cessation after a fixed period of 2 years in $\mathrm{MR}^{5}$, whereas $69 \%$ are expected to relapse. $^{43}$

Since the identification of patients who would benefit most from discontinuation of imatinib remains a key issue, the question of the duration of molecular response before discontinuation is crucial. One of the problems of most trials published recently is the unknown quality (that is, accuracy and sensitivity) of PCR measurements before study entry. In some studies even PCRs within the trial were not performed according to the IS, for example, in the ISAV trial. In addition, some patients were on imatinib treatment $>15$ years but standardization of laboratories has taken place only within the last 10 years. ${ }^{44}$ Therefore, the individual duration of $\mathrm{MR}^{4} / \mathrm{MR}^{4.5}$ and even MMR cannot be defined accurately.

\section{Sokal score}

Data from the STIM trial suggest that the probability of remaining in stable MR after discontinuation is more favorable in the low-risk group (according to the Sokal Score) than in the intermediate or high-risk groups. Using multivariate analysis and logistic regression at 8 months, Sokal risk and imatinib therapy duration were confirmed as two independent prognostic factors for prediction of molecular relapse after imatinib cessation. This was confirmed very recently. ${ }^{24,25}$ Using univariate analysis, Ross et al. ${ }^{26}$ in the TWISTER study found that high-risk Sokal score at diagnosis was the strongest predictor of molecular recurrence after stopping treatment. It is of some interest to note that a factor like the Sokal score illustrating the aggressiveness of the disease at diagnosis is still significant.

\section{Safety}

More than $2000 \mathrm{CML}$ patients have ceased treatment after achieving deep molecular response. So far, all patients who have relapsed remained sensitive to TKI re-treatment and no patient has developed BCR-ABL mutations.

To the best of our knowledge only one case of progression to blast crisis has been reported in a patient who stopped treatment in A-STIM. This patient was retreated with imatinib after molecular relapse and regained molecular response. However, the patient then experienced a lymphoid blast crisis 8.5 months later. The patient was treated with nilotinib and chemotherapy, and then underwent a successful allogeneic stem cell transplantation. ${ }^{30}$

\section{IMPACT OF STOPPING CML THERAPY ON PATIENTS}

\section{Patient-centered}

The life expectancy of CML patients is now very close to that of the healthy population. For this reason, the improvement of patient health and well-being has become the main goal of CML management, a development that could not have been foreseen 10 years ago. Therefore, a primary motivating factor for TFR initiation is the desire and expectation of patients to lead a normal life. For CML patients the QoL must be considered normal or close to normal. Obviously, a reduction in the side effects associated with continuous TKI therapy is a primary consideration. It seems that the QoL in CML patients is lower than that of the normal population especially in younger patients. ${ }^{7,45}$ Patient perspectives are a very important and sometimes an overlooked aspect of CML management. A recent survey ${ }^{46}$ found that not all patients are willing to stop therapy. The survey also found that, for patients 


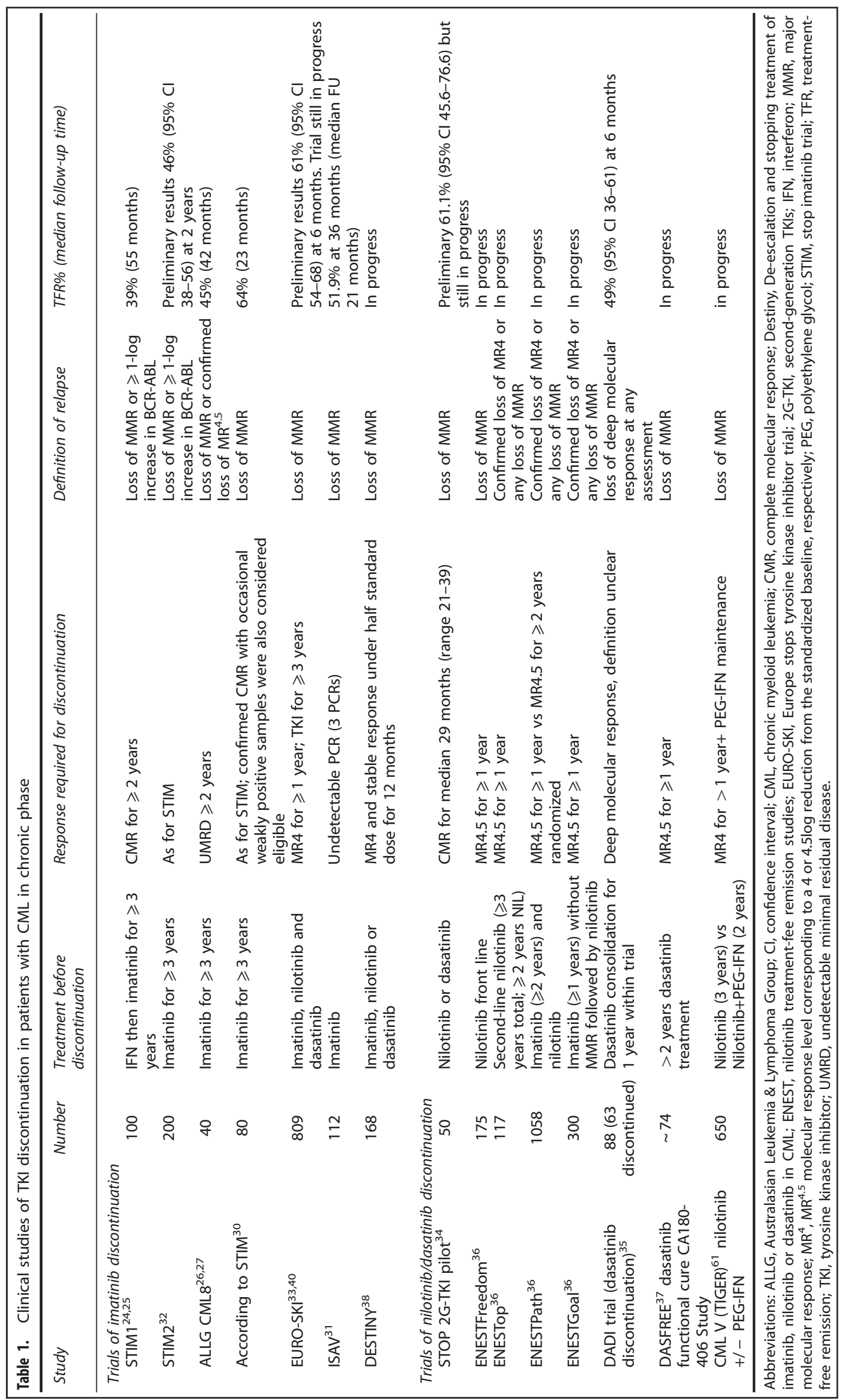


who would consider stopping therapy, the motivation is associated with cost and age.

A physician cannot practice today without having a global awareness of the price of drugs and taking into consideration that reducing economic burden is necessary to treat all patients worldwide. In many places, reduced cost is a motivating factor for TFR initiation. However, as generic versions of imatinib become more widely available, the costs of the increased monitoring for those on TFR may be comparatively more expensive than continued treatment, at least with generic imatinib. An interesting factor is that in many countries comprehensive health insurance means that reducing the cost of treatment is not usually a patient driven consideration, but may be a health payer-driven goal. ${ }^{47}$

\section{Side effects of TKI discontinuation}

Although imatinib and other TKIs can induce side effects in the musculoskeletal system, it has been assumed that such adverse events are reversible on cessation of therapy. However, in the ongoing EURO-SKI trial, some patients reported musculoskeletal pain starting or worsening 1-6 weeks after STIM therapy. This was more specifically investigated in a sub-cohort of the trial where it occurred in 15 out of 50 of patients. ${ }^{48}$ The pain was localized to various parts of the body, including the shoulder and hip regions and/or extremities, sometimes resembling polymyalgia rheumatica. Only very minor laboratory abnormalities were noted in association with the musculoskeletal symptoms. Symptoms were mild in most individuals, leading only to use of non-prescription drugs (paracetamol or other non-stereoidal antiinflammatory drugs, non-steroidal anti-inflammatory drugs), but some were more severely afflicted with manifestations interfering with everyday activities and requiring steroid therapy. Over time these symptoms seem to resolve. Rate of molecular relapse in patients with musculoskeletal pain did not differ from those without these symptoms.

Similar observations were made in a cessation trial conducted in Korea where $55 \mathrm{CML}$ patients were requested to fill out a QoL questionnaire and $22 \%$ of the patients reported onset of or worsening of musculoskeletal pain after stopping imatinib. ${ }^{49}$ Also in the ISAV study mentioned above and in the STIM2 trial, $21 \%$ of the patients reported musculoskeletal symptoms compatible with this 'TKI-withdrawal syndrome'. ${ }^{31,50}$

The mechanism underlying the 'TKI-withdrawal syndrome' is unknown. It is possible that targets of TKIs other than BCR-ABL such as KIT and or PDGFR are involved in this phenomenon. ${ }^{51}$ In a small study in Italy, two out of seven patients stopping imatinib reported grade 2 musculoskeletal pain developing 2-3 weeks after TKI cessation; in both of these patients an increased level of PDGFbeta in plasma was found. ${ }^{52}$

It seems that this phenomenon is not restricted to imatinib pretreatment. Physicians should be aware of the possibility of adverse events appearing after stopping long-term TKI therapy and promptly report such symptoms. Further investigations into underlying mechanisms are also warranted.

\section{WHY IS TKI CESSATION RESTRICTED TO A MINORITY OF CML PATIENTS?}

There are two factors that must be taken into consideration in the interpretation of the TFR results: first, the biology of the leukemic stem cells which have been reported resistant to TKI, and, second, the immune control of the disease provided by the results of allogeneic transplantation or indirectly from IFN-alpha treatment era.

Several studies have shown that $\mathrm{Ph}+$ hematopoietic stem cells (CD34+/CD38 - cells) are not sensitive to TKls as these cells are not dependent on BCR-ABL1 for their survival, at least not in short term in vitro cultures. ${ }^{53,54}$ Based solely on this observation, one would expect relapse in virtually all patients after TKI discontinuation. However, as documented in this review stopping TKI therapy is clinically feasible in $\sim 40-60 \%$ of patients with deep molecular response.

A major question in the field has become whether TKI-therapy in vivo, as opposed to the in vitro findings cited above, over time results in elimination of CML stem cells and, furthermore, whether this is a requirement for long-term TFR after TKI cessation. Two individual studies have shown that, in CML patients treated with imatinib for many years and in whom routine qRT-PCR shows no detectable $B C R-A B L$, evidence of the disease persists. This was demonstrated either by the existence of $\mathrm{Ph}+$ long-term culture initiating cells $(\mathrm{LSCs})^{55}$ or as BCR-ABL expressing sorted CD34 +/CD38-negative cells. ${ }^{56}$ In addition, using patient-specific genomic PCR analysis, Ross et al. ${ }^{27}$ demonstrated that BCR-ABL can be detected at the genomic level for many years in patients in TFR.

In summary, these findings indicate that CML stem cells may persist even after prolonged TKI therapy and that elimination of these cells are not necessary in all patients for lasting TFR. It also indicates that low levels of disease in some patients may be controlled by mechanisms not yet fully revealed. However, targeting stem cells is still of major interest in preclinical and clinical research, and potential stem-cell active compounds are under investigation. So far, the transfer into clinical practice is pending. ${ }^{57}$

Fluctuating BCR-ABL levels detected by qRT-PCR just below MMR without loss of MMR (and thus without re-treatment) is a common finding in stopping trials, as described, for example, in the previously mentioned A-STIM study. ${ }^{30}$ This suggests the importance of the role of immune surveillance for sustained TFR in CML.

During the IFN era, the immune system was identified as an important factor in the response to IFN. Its pleiotropic mechanism of action in CML includes immune activation and specific targeting of CML stem cells, ${ }^{58}$ especially by inducing long-term survival of cytotoxic T cells, enhancing natural killer (NK) cell cytotoxic activity and differentiating of dendritic cells. ${ }^{59}$

These effects may also be important for the maintenance of TFR after stopping treatment in CML whether or not IFN was within the treatment schedule or IFN was used as a maintenance therapy. Pilot data were positive, ${ }^{60}$ but there is strong need to investigate this important question within well-designed trials, for example, the CML V (TIGER) trial which compares the efficacy of nilotinib alone with nilotinib plus IFN-alpha as a first-line therapy. ${ }^{61}$ A stopping procedure is integrated in both arms to evaluate the role of IFN alpha in the context of treatment-free remission.

In support of the importance of the immune system for TFR, two independent studies have reported that low NK-cell numbers may predict early disease relapse after TKI discontinuation. ${ }^{62-65}$ These observations suggest that NK-cell-based immune surveillance may contribute to CML control after TKI cessation.

Whether NK-cell number and function may be used among other factors to predict disease relapse after TKI discontinuation needs to be further investigated. In one of the studies, NK cell numbers were significantly different in early relapses $(\leqslant 5$ months after TKI stop) vs late relapses ( $>5$ months after TKI stop). Thus, different mechanisms may be involved in return of the disease at different time points. It further remains to be determined if pharmacological use of agent(s) that stimulate NK-cell function can increase the number of CML patients achieving deep molecular response and long-term TFR after TKI cessation.

Recently, Caocci et al. ${ }^{66}$ reported KIR polymorphisms that could be significantly associated with better results of TFR. They published a cohort of $36 \mathrm{CP}-\mathrm{CML}$ patients who discontinued TKI therapy and found using multivariate analysis that TFR was significantly higher in patients homozygous for KIR A/A haplotype vs $\mathrm{KIR} \mathrm{B} / \mathrm{x}$ haplotype (85.7 vs $45.5 \% ; P=0.029$ ). One of the 
haplotypes has already been reported to be significantly linked to the TKI response (KIR2DL5B). ${ }^{67}$

If molecular recurrence after stopping TKI is due to the persistence of LSCs, we need to understand why quiescent LSCs are insensitive to TKIs. This question has been addressed in a large number of publications focused on targeting LSCs. ${ }^{57}$ This strategy is based on the fact that, in comparison to normal stem cells, LSCs exhibit aberrant or non-regulated self-renewal, survival and dormancy. Several strategies have been proposed to target LSCs including inhibiting survival/renewal pathways, sensitizing LSC (cycling or differentiating), immune targeting or modifying the bone marrow niche. JAK/STAT, JAK2 kinase, the protein phosphatase 2A (PP2A), arachidonate 5-lipoxygenase gene (ALOX5), histone deacetylases (HDACs), sirtuin 1 (SIRT1) and BCL6 are among the most relevant targets for such a strategy. Two of the most important pathways for self-renewal of CML LSCs are the Wnt/beta-catenin and the Hedgehog pathways. ${ }^{57,68}$

A recent study demonstrated that it is possible to induce a kind of erosion of leukemic stem cells using a PPARgamma agonist with a drug currently used for diabetes therapy. The main mechanism is supported by a reduction of STAT5 activity to regulate the pathway that controls quiescence in LSCs, causing the gradual erosion of this cellular pool. ${ }^{69}$

\section{Why is TKI discontinuation still experimental?}

As summarized in this review, a substantial number of studies on TKI cessation have been published or are ongoing. Limitations of the hitherto published trials are the relatively small patient cohorts, the unproven prognostic factors, the lack of standardized definition of molecular response, especially before study entry but even within studies, as well as the heterogeneous criteria used for stopping treatment and initiating re-treatment after relapse.

\section{Definition of molecular response}

The depth of response is an important factor in the decision to discontinue TKI treatment. The definition of molecular response and the standardization of $B C R-A B L$ transcript measurement remain a concern. The expression of the control gene used is critically important as it determines the sensitivity of the result independent of the $B C R-A B L$ detection assay. ${ }^{70}$ For this reason, the CML Working Group of the ELN has recently proposed revised definitions of molecular response that take into account the sensitivity of the molecular test, that is, $\mathrm{MR}^{4}$ indicates $\geqslant 4$-log reduction $\left(B C R-A B L^{\text {IS }} \leqslant 0.01 \%\right), M^{4.5}$ indicates $\geqslant 4.5$-log reduction $\left(B C R-A B L^{1 S} \leqslant 0.0032 \%\right)$, and $M^{5}$ indicates $\geqslant 5$-log reduction (BCR$\left.A B L^{1 S} \leqslant 0.001 \%\right)$, especially in negative qRT-PCR results. ${ }^{15}$ Different European laboratories working in a European molecular network within the EUTOS program have validated this standardization. ${ }^{71}$ Consequently, terms like 'CMR' and 'undetectable minimal residual disease (UMRD)' should no longer be used, as the sensitivity of the assay is not clearly described. Table 1 describes the different molecular levels and definitions that have been used so far for inclusion in TKI cessation trials in CML.

Current qRT-PCR methods can reliably detect up to a 5-log reduction in $B C R-A B L$, but newer techniques, such as DNA-based PCR, RNA-based digital PCR and replicated PCR, have demonstrated increased biological and/or technical sensitivity and may enable the assessment of even deeper levels of molecular response. ${ }^{72}$ However, it should be noted that, in historic assays low level of BCR-ABL transcripts have been found in the blood of normal individuals. ${ }^{73,74}$ These findings were based on a large volume of peripheral blood investigated $(400 \mathrm{ml})$ and have not been confirmed with newer technologies. On the other hand, dormant CML stem cells may persist independent of BCR-ABL and express $B C R-A B L$ transcripts on a low level, which indicate the need for genomic assessment as compared with RNA-based technologies. $^{75}$
We still do not know the threshold of residual disease which will allow us to safely stop TKI with the lowest rate of molecular recurrence. In part, we will gain new knowledge from the ongoing studies, but, despite the attempts made in some of the ENEST trials and the CML V trial, the uncertainty of molecular results before study entry is a problem for all trials.

\section{Frequency of monitoring}

As shown in several trials, the need for re-initiating therapy is necessary within $6-7$ months in $\sim 45-60 \%$ of patients. To not miss the time point and to avoid a fast and steep increase in BCR-ABL transcripts, frequent monitoring is strongly recommended. In most of the recent studies, this was done 4-weekly, after 6 months follow-up 6-weekly. If BCR-ABL transcripts are stable, thereafter, 3-monthly monitoring seems to be safe. This procedure has to be individually adopted according to the level of fluctuating transcripts.

\section{Initiating re-treatment}

Because many studies on TKI cessation have been launched, the criteria of treatment re-challenge in the different trials must be outlined clearly (Table 1). In the STIM studies, molecular relapse was defined as positivity of BCR-ABL transcript in qRT-PCR confirmed by a second analysis point indicating the increase of $1 \mathrm{log}$ in relation to the first analysis point at two successive assessments, or as loss of MMR at one point. This definition led to the proposal of the term 'molecular recurrence' in place of 'molecular relapse'. ${ }^{76}$ In the TWISTER study, molecular relapse was defined as two consecutive positive samples at any value or loss of MMR. The results of STIM and TWISTER studies are very similar and comparable. ${ }^{24,26}$ The definition used in A-STIM was different from that used in the STIM study. Molecular relapse was less stringently defined than in STIM as loss of MMR at any time was chosen for triggering re-treatment. It was the A-STIM trial that established loss of MMR as a trigger for restarting TKI therapy in CP-CML patients who have stopped imatinib after achieving durable molecular response. It is precisely this criterion that was chosen in EURO-SKI and other ongoing studies to define molecular relapse.

What can be done to raise patient numbers for stopping attempts?

An important step in increasing the number of candidates for TKI discontinuation is to increase the percentage of patients in durable deep MR (MR ${ }^{4}, \mathrm{MR}^{4.5}$ or deeper) to be eligible for stopping trials.

Using the criteria of the STIM and TWISTER studies, it should be possible to predict which patients are ideal for discontinuation of TKIs. Recently, Branford et al. found in a study of 415 patients treated with imatinib for 8 years that the cumulative rate of stable $\mathrm{MR}^{4.5}$ (for at least 2 years) was $43 \%$. In these patients, the time to achieve MMR was correlated with the time to achieve stable $\mathrm{MR}^{4.5}{ }^{28}$ In addition, the only two independent factors, that is, female sex and a low level of BCR-ABL value at 3 months, were strongly statistically linked to the prediction of sustained $M R^{4.5}$. Factors associated with sustained $M^{4.5}$ and undetectable transcripts induced by TKI (imatinib, dasatinib and nilotinib) were also analyzed in a multivariate analysis $(N=495)$ by Falchi et al., ${ }^{77}$ and showed that older age, higher baseline hemoglobin, higher baseline platelets, TKI modality and response at 3 months were significant. A larger cohort of patients would be necessary to validate and refine this analysis. The German CML Study IV provides this cohort. In more than 1500 patients, the cumulative incidence of confirmed $\mathrm{MR}^{4.5}$ was $54 \%$ after 9 years. ${ }^{3}$ The number of patients at this time point is probably lower as cumulative incidence usually overestimates total amounts. The goal of CML IV has been to demonstrate the link between $\mathrm{MR}^{4.5}$ achievement and 
better survival. But identification of specific predictive factors of TFR must be deferred until the final analysis of ongoing trials has been carried out.

How best to increase the proportion of patients eligible for stopping approach?

If stopping CML treatment will be recommended in a well-defined patient population, the next step will be to think about how to increase patient numbers fulfilling these definitions. In this context, many factors have to be considered which are beyond this review, for example, optimizing first-line therapy, switching patients in MMR to other drugs to gain deeper MR. With the initial use of second-generation TKIs faster and deeper MR is achievable. $^{78-80}$ The specific impact on TFR should be validated and current first-line treatment strategies should be critically assessed. ${ }^{81}$ Further, the addition of other drugs, for example, IFN or stem cell active drugs, may also increase the proportion of candidates for cessation attempts.

\section{Pregnancy and children}

In this review, the specific situation of pregnancy has not been discussed. Depending on the individual situation of pregnant patients a variety of strategies should be considered. A detailed review on management of pregnancies in CML was published recently. ${ }^{82}$ If possible, we recommend that female patients at least fulfill criteria of stopping trials before conception. In addition, monitoring procedures should be adapted accordingly and eventually individually changed.

So far, no data for stopping treatment in children are published. It has been recommended to abstain from such an approach in children since the disease seems to be more aggressive. ${ }^{83}$

\section{RECOMMENDATIONS FOR CLINICAL PRACTICE 2016}

At present, definitive recommendations cannot be given. A primary reason for this is the discrepancy of definitions for stopping and re-initiating treatment in hitherto published studies. Nevertheless, despite the lack of consensus on the pre-requisite of molecular response and on the importance of the speed of achieving a deep response, we are able to formulate a few recommendations or rules for stopping treatment (Table 2). First of all, we strongly recommend the inclusion of patients in clinical trials whenever possible. Only patients in chronic phase with no history of failure according to ELN criteria should be considered for stopping. It is necessary to identify the type of BCR-ABL transcript at diagnosis to avoid false-negative $P C R$ results during follow-up. Achievement of a deep molecular response, at the least $\mathrm{MR}^{4}$, before stop is mandatory. This $\mathrm{MR}^{4}$ has to be maintained or sustained for some time. For instance, the pre-requisite of an $M^{4}{ }^{4}$ or lower response for 2 years seems reasonable as it suggests that treating patients for several years induces a kind of exhaustion of the disease. Patients must receive follow-up in a center where standardized molecular biology is performed using IS according to what has been agreed upon and published. ${ }^{16,84}$

\section{CONCLUSIONS}

CML is more than ever the model of targeted therapy for human malignancies. The success of imatinib and second-generation TKIs has dramatically changed the outcome for CML patients. As TKItreated CML patients have a near-normal life expectancy, two important issues have emerged that demand our consideration: (i) QoL and ethical aspects of the treatment and (ii) the economic impact of treating patients over the course of a normal lifespan.

One of the best ways to approach these two points is to consider the discontinuation of TKI in well-responding patients. Such a strategy has been proposed in several recent studies, and around the world more than 2000 patients have officially stopped TKI in clinical trials. However, many questions about depth of MR, duration of treatment, predictive factors and safety remain open, and are topics of continuing discussion.

The current ambition in CML treatment strategy is to develop a definition of TKI discontinuation in CML patients with a view to its implementation as a standard treatment protocol in clinical practice. Well-defined prognostic factors, standardized molecular monitoring and further characterization of successful treatment stop with the help of well-designed scientific studies in clinical trials are necessary for identifying those patients on whom the TKI discontinuation procedure may be safely applied.

Table 2. Features of CML and TKI therapy that guide attempts to stop therapy

\begin{tabular}{ll}
\hline Feature & Comment \\
\hline $\begin{array}{l}\text { Chronic phase disease } \\
\text { Transcript type }\end{array}$ & $\begin{array}{l}\text { Patients with history of advanced phase disease or failure to TKI treatment have a higher risk of relapse. } \\
\text { Only patients with known typical BCR-ABL transcript type at diagnosis should be considered for } \\
\text { stopping to secure appropriate follow-up monitoring. }\end{array}$ \\
$\begin{array}{l}\text { Sokal score } \\
\text { Line of treatment }\end{array}$ & $\begin{array}{l}\text { Patients on first-line TKI therapy and those on second line due to intolerance to first line may be } \\
\text { considered for a cessation attempt. }\end{array}$ \\
Duration of TKI therapy before & $\begin{array}{l}\text { Preliminary data suggest a favorable impact of a longer duration of therapy, but exact numbers are } \\
\text { pending. Ongoing studies like EURO-SKI will determine optimal duration of therapy before a stop }\end{array}$ \\
stop & $\begin{array}{l}\text { attempt may be considered. } \\
\text { Level of MR before stop }\end{array}$ \\
Duration of deep MR before stop & $\begin{array}{l}\text { Letermine level and duration of MR before a stop attempt may be considered. } \\
\text { will determine level and duration of MR before a stop attempt may be considered. }\end{array}$ \\
Molecular monitoring & $\begin{array}{l}\text { The PCR-monitoring schedule used in EURO-SKI, 4-weekly for } 6 \text { months, every } 6 \text { weeks months 7-12 and } \\
\text { then every } 3 \text { months appears to be safe and practical considering current data. }\end{array}$ \\
Role of maintenance therapy with & Exact role has not been defined yet. Prospective trials are ongoing. \\
IFN after TKI stop & Loss of MMR at one point as used in A-STIM and EURO-SKI seems to be safe and practical considering \\
Definition of molecular relapse & current evidence. \\
and trigger for restart of therapy &
\end{tabular}

Abbreviations: A-STIM, according to stop imatinib; CML, chronic myeloid leukemia; EURO-SKI, Europe stops tyrosine kinase inhibitor trial; IFN, interferon; MMR, major molecular response; $\mathrm{MR}^{4}, \mathrm{MR}^{4.5}$ molecular response level corresponding to a 4 or 4.5 log reduction from the standardized baseline, respectively; PEG, polyethylene glycol; TKI, tyrosine kinase inhibitor. 
Trials are needed that will enable identification of patients who possibly have been cured of their disease by a long course of TKI treatment. Mechanisms responsible for maintenance of deep MR off TKI treatment must be unraveled. In turn, this will pave the way for new therapeutic strategies that will eventually lead to high cure rates in CML. Furthermore, such studies should (1) address optimized treatments, (2) identify prognostic markers to better predict response behavior of $\mathrm{CML}$ patients with regard to persistence of MR after TKI discontinuation and (3) compare QoL before and after TKI discontinuation.

In conclusion, the impact of stopping strategies in CML will be enormous for patients, health care systems and society at large. Studies are needed which will guide physicians in determining when it is safe and most promising to stop TKI therapy in CML patients. This will have a large economic impact on CML treatment. With the increasing prevalence of CML patients and the high costs of TKI treatment per year, stopping treatment in CML will result in a considerable and durable reduction of treatment costs worldwide. The important question of how to increase the proportion of patients is being addressed by treatment optimization studies.

We expect that this new treatment strategy will have a major impact on the understanding of the determinants involved in the cure of CML.

\section{CONFLICT OF INTEREST}

SS received honoraria from Novartis, Bristol-Myers Squibb (BMS), Pfizer, ARIAD and research funding from Novartis and BMS; JR research support and/or honoraria by Novartis, BMS, Pfizer and Ariad; AH research support and honoraria by Novartis, BMS, Pfizer and Ariad, and FXM research support and/or honoraria by Novartis, BMS, Pfizer and Ariad.

\section{REFERENCES}

1 Gambacorti-Passerini C, Antolini L, Mahon FX, Guilhot F, Deininger M, Fava C et al. Multicenter independent assessment of outcomes in chronic myeloid leukemia patients treated with imatinib. J Natl Cancer Inst 2011; 103: 553-561.

2 Bjorkholm M, Ohm L, Eloranta S, Derolf A, Hultcrantz M, Sjoberg J et al. Success Story of targeted therapy in chronic myeloid leukemia: a population-based study of patients diagnosed in Sweden from 1973 to 2008. J Clin Oncol 2011; 29: 2514-2520.

3 Hehlmann R, Müller MC, Lauseker M, Hanfstein B, Fabarius A, Schreiber A et al. Deep molecular response is reached by the majority of patients treated with imatinib, predicts survival, and is achieved more quickly by optimized high-dose imatinib: results from the randomized CML-study IV. J Clin Oncol 2014; 32: 415-423.

4 Castagnetti F, Gugliotta G, Breccia M, Stagno F, lurlo A, Albano F et al. Long-term outcome of chronic myeloid leukemia patients treated frontline with imatinib. Leukemia 2015; 29: 1823-1831.

5 National Comprehensive Cancer Network. Chronic Myelogenous Leukemia. NCCN Clinical Practice Guidelines in Oncology. 2012.

6 Baccarani M, Deininger MW, Rosti G, Hochhaus A, Soverini S, Apperley JF et al. European LeukemiaNet recommendations for the management of chronic myeloid leukemia: 2013. Blood 2013; 122: 872-884.

7 Efficace F, Baccarani M, Breccia M, Alimena G, Rosti G, Cottone F et al. Health-related quality of life in chronic myeloid leukemia patients receiving longterm therapy with imatinib compared with the general population. Blood 2011; 118: 4554-4560.

8 Kalmanti L, Saussele S, Lauseker M, Muller MC, Dietz CT, Heinrich L et al. Safety and efficacy of imatinib in $\mathrm{cml}$ over a period of 10 years: data from the randomized cml-study iv. Leukemia 2015; 29: 1123-1132.

9 Cortes JE, Kim DW, Pinilla-lbarz J, le Coutre P, Paquette R, Chuah C et al. A phase 2 trial of ponatinib in Philadelphia chromosome-positive leukemias. $N$ Engl J Med 2013; 369: 1783-1796.

10 Kim TD, Rea D, Schwarz M, Grille P, Nicolini FE, Rosti G et al. Peripheral artery occlusive disease in chronic phase chronic myeloid leukemia patients treated with nilotinib or imatinib. Leukemia 2013; 27: 1316-1321.

11 Efficace F, Baccarani M, Breccia M, Cottone F, Alimena G, Deliliers GL et al. Chronic fatigue is the most important factor limiting health-related quality of life of chronic myeloid leukemia patients treated with imatinib. Leukemia 2013; 27: 1511-1519.
12 Efficace F, Breccia M, Saussele S, Kossak U, Caocci G, Sprangers M et al. International development of an EORTC measure to assess patient-reported quality of life (QoL) and symptoms in chronic myeloid leukemia (CML). Blood 2011; 118: 1353-1354.

13 Lütge I, Pfirrmann M, Stalljann I, Illmer T, Schafhausen P, Kiani A et al. Healthrelated quality of life in CML patients under nilotinib firts-line treatment: Results of a German sub-study within the ENEST 1st trial. Hamatologica 2015; 100, Abstract P233.

14 Hochhaus A. Educational session: managing chronic myeloid leukemia as a chronic disease. Hematology Am Soc Hematol Educ Program 2011; 2011: 128-135.

15 Cross NCP, White HE, Müller MC, Saglio G, Hochhaus A. Standardized definitions of molecular response in chronic myeloid leukemia. Leukemia 2012; 26: 2172-2175.

16 Cross NC, Hochhaus A, Müller MC. Molecular monitoring of chronic myeloid leukemia: principles and interlaboratory standardization. Ann Hematol 2015; 94: S219-S225.

17 Mahon FX, Delbrel X, Cony-Makhoul P, Faberes C, Boiron JM, Barthe C et al. Follow-up of complete cytogenetic remission in patients with chronic myeloid leukemia after cessation of interferon alfa. J Clin Oncol 2002; 20: 214-220.

18 Kantarjian HM, O'Brien S, Cortes JE, Shan J, Giles FJ, Rios MB et al. Complete cytogenetic and molecular responses to interferon-alpha-based therapy for chronic myelogenous leukemia are associated with excellent long-term prognosis. Cancer 2003; 97: 1033-1041.

19 Cortes J, O'Brien S, Kantarjian H. To the editor: discontinuation of imatinib therapy after achieving a molecular response. Blood 2004; 104: 2204-2205.

20 Mauro MJ, Druker BJ, Maziarz RT. Divergent clinical outcome in two CML patients who discontinued imatinib therapy after achieving a molecular remission. Leuk Res 2004; 28: S71-S73.

21 Merante S, Orlandi E, Bernasconi P, Calatroni S, Boni M, Lazzarino M. Outcome of four patients with chronic myeloid leukemia after imatinib mesylate discontinuation. Haematologica 2005; 90: 979-981.

22 Rousselot $\mathrm{P}$, Huguet $\mathrm{F}$, Rea D, Legros L, Cayuela JM, Maarek $\mathrm{O}$ et al. Imatinib mesylate discontinuation in patients with chronic myelogenous leukemia in complete molecular remission for more than 2 years. Blood 2007; 109: 58-60.

23 Mahon FX. Is going for cure in chronic myeloid leukemia possible and justifiable? Hematology Am Soc Hematol Educ Program 2012; 2012: 122-128.

24 Mahon FX, Rea D, Guilhot J, Guilhot F, Huguet F, Nicolini F et al. Discontinuation of imatinib in patients with chronic myeloid leukaemia who have maintained complete molecular remission for at least 2 years: the prospective, multicentre Stop Imatinib (STIM) trial. Lancet Oncol 2010; 11: 1029-1035.

25 Etienne G, Rea D, Guilhot J, Guilhot F, Huguet F, Legros L et al. Long-Term Followup of the French 1 Stop Imatinib Study (STIM1) in Chronic Myeloid Leukemia Patients. ASH Annual Meeting Abstracts 2015: Abstract No. 345.

26 Ross DM, Branford S, Seymour JF, Schwarer AP, Arthur C, Yeung DT et al. Safety and efficacy of imatinib cessation for CML patients with stable undetectable minimal residual disease: results from the TWISTER study. Blood 2013; 122: 515-522.

27 Ross DM, Branford S, Seymour JF, Schwarer AP, Arthur C, Bartley PA et al. Patients with chronic myeloid leukemia who maintain a complete molecular response after stopping imatinib treatment have evidence of persistent leukemia by DNA PCR. Leukemia 2010; 24: 1719-1724.

28 Branford S, Yeung DT, Ross DM, Prime JA, Field CR, Altamura HK et al. Early molecular response and female sex strongly predict stable undetectable BCRABL1, the criteria for imatinib discontinuation in patients with CML. Blood 2013; 121: 3818-3824.

29 Takahashi N, Kyo T, Maeda Y, Sugihara T, Usuki K, Kawaguchi T et al. Discontinuation of imatinib in Japanese patients with chronic myeloid leukemia. Haematologica 2012; 97: 903-906.

30 Rousselot $\mathrm{P}$, Charbonnier A, Cony-Makhoul $\mathrm{P}$, Agape $\mathrm{P}$, Nicolini FE, Varet $\mathrm{B}$ et al. Loss of major molecular response as a trigger for restarting tyrosine kinase inhibitor therapy in patients with chronic-phase chronic myelogenous leukemia who have stopped imatinib after durable undetectable disease. J Clin Oncol 2014; 32: 424-430.

31 Mori S, Vagge E, le Coutre P, Abruzzese E, Martino B, Pungolino E et al. Age and $\mathrm{dPCR}$ can predict relapse in $\mathrm{CML}$ patients who discontinued imatinib: the ISAV study. Am J Hematol 2015; 90: 910-914.

32 Nicolini FE, Noël M-P, Escoffre M, Charbonnier A, Rea D, Dubruille V et al. Preliminary report of the STIM2 study: a multicenter stop imatinib trial for chronic phase chronic myeloid leukemia de novo patients on imatinib. Blood 2013; 122: Abstract 654.

33 Saussele S, Richter J, Guilhot J, Müller M, Dietz C, Porkka K et al. First interim analysis of a Pan-European Stop trial using standardized molecular criteria: results of the EURO-SKI trial.19th Congress of the European Hematology Association, Milan, Italy, June 12-15, 2014. Haematologica 2014; 99: 792; Abstract LB2440. 
34 Rea D, Rousselot P, Guilhot F, Tulliez M, Nicolini FE, Guerci-Bresler A et al. Discontinuation of second generation (2G) tyrosine kinase inhibitors (TKI) in chronic phase $(\mathrm{CP})$-chronic myeloid leukemia $(\mathrm{CML})$ patients with stable undetectable BCR-ABL transcripts. ASH Annual Meeting Abstracts 2012; 120: 916.

35 Imagawa J, Tanaka H, Okada M, Nakamae H, Hino M, Murai K et al. Discontinuation of dasatinib in patients with chronic myeloid leukaemia who have maintained deep molecular response for longer than 1 year (DADI trial): a multicentre phase 2 trial. Lancet Haematol 2015; 2: e528-e535.

36 Mahon FX, Baccarani M, Mauro MJ, Hughes TP, Saglio G, Savona M et al. Treatment-free remission (TFR) following nilotinib (NIL) in patients (pts) with chronic myeloid leukemia in chronic phase (CML-CP): ENESTfreedom, ENESTop, ENESTgoal, and ENESTpath. J Clin Oncol 2014; 32: abstract TPS7124.

37 Bristol-Myers Squibb. Open-Label Study Evaluating Dasatinib Therapy Discontinuation in Patients With Chronic Phase Chronic Myeloid Leukemia With Stable Complete Molecular Response (DASFREE). In: ClinicalTrials.gov [Internet]. Bethesda (MD):National Library of Medicine (US). Available at https://clinicaltrials. gov/ct2/show/NCT01850004 NLM Identifier: NCT01850004. 2015.

38 University of Liverpool. A Trial of De-escalation and Stopping Treatment in Chronic Myeloid Leukaemia Patients With Excellent Responses to Tyrosine Kinase Inhibitor Therapy (DESTINY). In: ClinicalTrials.gov [Internet]. Bethesda (MD): National Library of Medicine (US). Available at: https://clinicaltrials.gov/show/ NCT01804985 NLM Identifier: NCT01804985 2015.

39 Esteve J, Benhamou E, Raymond L. Statistical methods in cancer research. Volume IV. Descriptive epidemiology. IARC Sci Publ 1994; IV: 1-302.

40 Mahon FX, Richter J, Guilhot J, Muller MC, Dietz C, Porkka K et al. Interim Analysis of a Pan European Stop Tyrosine Kinase Inhibitor Trial in Chronic Myeloid Leukemia: The EURO-SKI study. Blood 2014; 124: 151

41 Stein AM, Bottino D, Modur V, Branford S, Kaeda J, Goldman JM et al. BCR-ABL transcript dynamics support the hypothesis that leukemic stem cells are reduced during imatinib treatment. Clin Cancer Res 2011; 17: 6812-6821.

42 Roeder I, Horn M, Glauche I, Hochhaus A, Mueller MC, Loeffler M. Dynamic modeling of imatinib-treated chronic myeloid leukemia: functional insights and clinical implications. Nat Med 2006; 12: 1181-1184.

43 Horn M, Glauche I, Muller MC, Hehlmann R, Hochhaus A, Loeffler M et al. Modelbased decision rules reduce the risk of molecular relapse after cessation of tyrosine kinase inhibitor therapy in chronic myeloid leukemia. Blood 2013; 121: 378-384.

44 Hughes T, Deininger M, Hochhaus A, Branford S, Radich J, Kaeda J et al. Monitoring $\mathrm{CML}$ patients responding to treatment with tyrosine kinase inhibitors: review and recommendations for harmonizing current methodology for detecting $B C R-A B L$ transcripts and kinase domain mutations and for expressing results. Blood 2006; 108: 28-37.

45 Saussele S, Stein M, Gil A, Kossak-Roth U, Lauseker M, Proetel U et al. Health-Related Quality of Life Impairment in Patients with Chronic Myeloid Leukemia: Results of a German Cross-Sectional Study of Patients Registered in Prospective, Controlled Clinical Trials. ASH Annual Meeting Abstracts 2015: Abstract No. 2773.

46 Goldberg S, Hamarman S. Patients with Chronic Myelogenous Leukemia May Not Want to Discontinue Tyrosine Kinase Inhibitor Therapy. ASH Annual Meeting Abstracts 2015: Abstract No. 1584.

47 Experts in Chronic Myeloid L. The price of drugs for chronic myeloid leukemia $(\mathrm{CML})$ is a reflection of the unsustainable prices of cancer drugs: from the perspective of a large group of CML experts. Blood 2013; 121: 4439-4442.

48 Richter J, Soderlund S, Lubking A, Dreimane A, Lotfi K, Markevarn B et al. Musculoskeletal pain in patients with chronic myeloid leukemia after discontinuation of imatinib: a tyrosine kinase inhibitor withdrawal syndrome? J Clin Oncol 2014; 32: 2821-2823.

49 Park JS, Lee SE, Jeong SH, Jang EJ, Choi MY, Kim HJ et al. Change of health-related profiles after Imatinib cessation in chronic phase chronic myeloid leukemia patients. Leuk Lymphoma 2016; 57: 341-347.

50 Berger MG, Pereira P, Oris C, Saugues S, Cony-Makhoul P, Gardembas M et al. Osteoarticular Pain after Discontinuation of Tyrosine Kinase Inhibitors (TKI): A French Cohort. ASH Annual Meeting Abstracts 2015: Abstract No. 137.

51 Buchdunger E, Cioffi CL, Law N, Stover D, Ohno-Jones S, Druker BJ et al. Abl protein-tyrosine kinase inhibitor STI571 inhibits in vitro signal transduction mediated by c-kit and platelet-derived growth factor receptors. J Pharmacol Exp Ther 2000; 295: 139-145.

52 Galimberti S, Fontanelli G, Barsotti S, Ricci F, Guerrini F, Ciabatti E et al. PDGFRB sustains the "withdrawal syndrome" after imatinib discontinuation. haematologica 2015; 100: 697 (abstract PB1759).

53 Corbin AS, Agarwal A, Loriaux M, Cortes J, Deininger MW, Druker BJ. Human chronic myeloid leukemia stem cells are insensitive to imatinib despite inhibition of BCR-ABL activity. J Clin Invest 2011; 121: 396-409.
54 Hamilton A, Helgason GV, Schemionek M, Zhang B, Myssina S, Allan EK et al. Chronic myeloid leukemia stem cells are not dependent on Bcr-Abl kinase activity for their survival. Blood 2012; 119: 1501-1510.

55 Chomel JC, Bonnet ML, Sorel N, Bertrand A, Meunier MC, Fichelson S et al. Leukemic stem cell persistence in chronic myeloid leukemia patients with sustained undetectable molecular residual disease. Blood 2011; 118: 3657-3660.

56 Chu S, McDonald T, Lin A, Chakraborty S, Huang Q, Snyder DS et al. Persistence of leukemia stem cells in chronic myelogenous leukemia patients in prolonged remission with imatinib treatment. Blood 2011; 118: 5565-5572.

57 Holyoake TL, Helgason GV. Do we need more drugs for chronic myeloid leukemia? Immunol Rev 2015; 263: 106-123.

58 Talpaz M, Mercer J, Hehlmann R. The interferon-alpha revival in CML. Ann Hematol 2015; 94: S195-S207.

59 Kanodia S, Wieder E, Lu S, Talpaz M, Alatrash G, Clise-Dwyer K et al. PR1-specific $T$ cells are associated with unmaintained cytogenetic remission of chronic myelogenous leukemia after interferon withdrawal. PLoS One 2010; 5: e11770.

60 Burchert A, Saussele S, Eigendorff E, Muller MC, Sohlbach K, Inselmann S et al. Interferon alpha 2 maintenance therapy may enable high rates of treatment discontinuation in chronic myeloid leukemia. Leukemia 2015; 29: 1331-1335.

61 University of Jena. Tasigna and Interferon Alpha Evaluation Initiated by the German Chronic Myeloid Leukemia Study Group - the TIGER Study (TIGER). In: ClinicalTrials.gov [Internet]. Bethesda (MD): National Library of Medicine (US). Available at https://clinicaltrials.gov/ct2/show/NCT01657604 NLM Identifier: NCT01657604; 2014

62 Ilander MM, Olsson-Strömberg U, Lähteenmäki H, Kasanen T, Koskenvesa $P$, Söderlund $S$ et al. Disease Relapse After TKI Discontinuation In CML Is Related Both To Low Number and Impaired Function Of NK-Cells:Data From Euro-SKI. Blood (ASH Annual Meeting Abstracts) 2013; 122: abstract 379.

63 Hughes A, Tang C, Clarson J, Vidovic L, Hughes TP, Yong AS Chronic Myeloid Leukemia Patients with Deep Molecular Responses to Tyrosine Kinase Inhibitors Have Increased Effector Natural Killer and Cytotoxic T Cell Immune Responses to Leukaemia-Associated Antigens and Concomitant Reduced Immune Suppressors. Blood (ASH Annual Meeting Abstracts) 2015; 126: abstract no. 18.

64 Ilander M, Schlums H, Olsson-Strömberg U, Lähteenmäki H, Kasanen T, Koskenvesa $P$ et al. Mature, Adaptive-like CD56DIM NK Cells in Chronic Myeloid Leukemia Patients in Treatment Free Remission. Blood (ASH Annual Meeting Abstracts) 2015; 126: abstract no. 343.

65 Burchert A, Inselmann S, Saussele S, Dietz CT, Müller MC, Eigendorff E et al. Frequency of CTLA-4 Receptor Ligand (CD86, B7.2) -Positive Plasmacytoid Dendritic Cells Predicts Risk of Disease Recurrence after Tyrosine-Kinase Inhibitor Discontinuation in Chronic Myeloid Leukemia: Results from a Prospective Substudy of the Euroski Trial. Blood (ASH Annual Meeting Abstracts) 2015; 126: abstract no. 599 .

66 Caocci G, Martino B, Greco M, Abruzzese E, Trawinska MM, Lai S et al. Killer immunoglobulin-like receptors can predict TKI treatment-free remission in chronic myeloid leukemia patients. Exp Hematol 2015; 43: 1015-1018.

67 Yeung DT, Vidovic L, Tang C, White DL, Branford S, Hughes TP et al. KIR2DL5B genotype independently predicts poor outcomes in CML-CP patients switched to nilotinib after suboptimal responses to imatinib and may refine prognosis in patients with EMR failure. Blood 2014; 124: 814-814.

68 Copland M. Chronic myelogenous leukemia stem cells: what's new? Curr Hematol Malig Rep 2009; 4: 66-73.

69 Prost S, Relouzat F, Spentchian M, Ouzegdouh Y, Saliba J, Massonnet G et al. Erosion of the chronic myeloid leukaemia stem cell pool by PPARgamma agonists. Nature 2015; 525: 380-383.

70 Deininger MW. Molecular monitoring in CML and the prospects for treatment-free remissions. Hematology Am Soc Hematol Educ Program 2015; 2015: 257-263.

71 Müller MC, Cross NC, Erben P, Schenk T, Hanfstein B, Ernst T et al. Harmonization of molecular monitoring of CML therapy in Europe. Leukemia 2009; 23: 1957-1963.

72 Melo JV, Ross DM. Minimal residual disease and discontinuation of therapy in chronic myeloid leukemia: can we aim at a cure? Hematology Am Soc Hematol Educ Program 2011; 2011: 136-142.

73 Biernaux C, Loos M, Sels A, Huez G, Stryckmans P. Detection of major bcr-abl gene expression at a very low level in blood cells of some healthy individuals. Blood 1995; 88: 3118-3122.

74 Bose S, Deininger M, Gora-Tybor J, Goldman JM, Melo JV. The presence of typical and atypical BCR-ABL fusion genes in leukocytes of normal individuals: biologic significance and implications for the assessment of minimal residual disease. Blood 1998; 92: 3362-3367.

75 Kumari A, Brendel C, Hochhaus A, Neubauer A, Burchert A. Low BCR-ABL expression levels in hematopoietic precursor cells enable persistence of chronic myeloid leukemia under imatinib. Blood 2012; 119: 530-539.

76 Deininger M. Hematology: curing CML with imatinib--a dream come true? Nat Rev Clin Oncol 2011; 8: 127-128. 
77 Falchi L, Kantarjian HM, Wang X, Verma D, Quintas-Cardama A, O'Brien S et al. Significance of deeper molecular responses in patients with chronic myeloid leukemia in early chronic phase treated with tyrosine kinase inhibitors. Am J Hematol 2013; 88: 1024-1029.

78 Hochhaus A, Saglio G, Hughes TP, Larson RA, Kim DW, Issaragrisil S et al. Longterm benefits and risks of frontline nilotinib vs imatinib for chronic myeloid leukemia in chronic phase: 5-year update of the randomized ENESTnd trial. Leukemia 2016; 30: 1044-1054.

79 Hochhaus A, Rosti G, Cross NC, Steegmann JL, le Coutre P, Ossenkoppele G et al. Frontline nilotinib in patients with chronic myeloid leukemia in chronic phase: results from the European ENEST1st study. Leukemia 2015; 30: 57-64.

80 Cortes JE, Saglio G, Kantarjian HM, Baccarani M, Mayer J, Boqué C et al. Final 5-year study results of DASISION: the DASatinib versus imatinib study in treatment-naive CML patients trial. J Clin Oncol 2016. accepted for publication on 22 March 2016.

81 Larson RA. Is there a best TKI for chronic phase CML? Blood 2015; 126: 2370-2375.
82 Palani R, Milojkovic D, Apperley JF. Managing pregnancy in chronic myeloid leukaemia. Ann Hematol 2015; 94: S167-S176.

83 Hijiya N, Schultz KR, Metzler M, Millot F, Suttorp M. Pediatric chronic myeloid leukemia is a unique disease that requires a different approach. Blood 2016; 127: 392-399.

84 Cross NC. Standardisation of molecular monitoring for chronic myeloid leukaemia. Best Pract Res Clin Haematol 2009; 22: 355-365.

(i) This work is licensed under a Creative Commons Attributioncc) NonCommercial-ShareAlike 4.0 International License. The images or other third party material in this article are included in the article's Creative Commons license, unless indicated otherwise in the credit line; if the material is not included under the Creative Commons license, users will need to obtain permission from the license holder to reproduce the material. To view a copy of this license, visit http:// creativecommons.org/licenses/by-nc-sa/4.0/ 